\title{
An industrial light field camera applied for 3D velocity measurements in flow past confined cylinder
}

\author{
Alexander Seredkin ${ }^{1,2, *}$, Maxim Shestakov ${ }^{1}$, and Mikhail Tokarev ${ }^{1}$ \\ ${ }^{1}$ Kutateladze Institute of Thermophysics, Siberian Branch of Russian Academy of Sciences 630090, \\ Novosibirsk, Akademika Lavrentieva Avenue, 1, Russia \\ ${ }^{2}$ Novosibirsk State University, 630090, Novosibirsk, st. Pirogova, 2, Russia
}

\begin{abstract}
Light field cameras have some advantages over classic cameras in a narrow field of application, such photography, cinematography surveillance and quality control in industry. Light field cameras have larger depth of field, compare to regular camera, but lower spatial resolution along optical axis then binocular system. However, 3D velocity measurements using light field are reasonable alternative to modern 3D PIV measurements. The nature of light field image requires extra reconstruction step which affects overall accuracy. In this article, light field PTV was compared to Tomo-PIV. Both techniques were used to measure $3 \mathrm{D}$ velocity fields in a turbulent wake past confined cylinder.
\end{abstract}

\section{Introduction}

Modern light-field optical devices have been started to use in experimental fluid dynamics research. Output images of the light-field cameras contain extra information which is used to get the third spatial coordinate. Several reconstruction algorithms for light field images have been proposed [1, 2, 3, 4]. In general, calculation of a depth map from a light field image is an ill-posed problem, since a target equation system is underdetermined, similar to $3 \mathrm{D}$ reconstruction from several $2 \mathrm{D}$ projections. To get a reasonable approximation of a solution some assumption on reconstructed objects of research should be done. The better results require compliance between the object of study, camera settings and reconstruction algorithms.

In the present work, experimental whole-field measurements of the velocity of fluid flow in a slotted jet [5] and the wake of a cylinder using a light field camera are performed. The experiment was carried out in a modified closed hydrodynamic test section [5] with a tank of constant level at $\mathrm{Re} \sim 3500$. The used Light-Field PTV reconstruction techniques, results of the experiment, and comparison of the results with a reference Tomo-PIV measurements are described below.

\footnotetext{
*Corresponding author: sasha7-93@ mail.ru
} 


\section{Methods}

In this section we describe main steps of the data processing. In the beginning an image of a uniform white background is captured to get microlens coordinates (see Fig. 1). Known microlens structure of the image allows performing a program refocus procedure. The program refocus procedure focuses a raw image on an arbitrary plane. In this case off-focus areas become blurred, similar to classic camera images. This procedure is convenient to convert an image from the light field model to a normal image.

A developed 3D reconstruction algorithm is based on a refocusing procedure. The structure of repeated images depends on a virtual depth and determines an actual depth value. If the virtual depth is correct, then the reconstructed image will be on focus. The idea of the proposed algorithm is to calculate the standard deviation between intensity values corresponding to an object point visualized by different microclenses to assess to the depth of the object point. The closer the obtained virtual depth to a true value, the lower the standard deviation is. An initial image is divided into small rectangular areas that are reconstructed independently. For each of these areas the algorithm finds a virtual depth that minimizes standard deviation value. The found virtual depths form a depth map for the registered object. Then refocusing algorithm reconstructs each part of the image according to the depth map. That patchwork image is called "total focus", since every element is in focus [5].

The virtual depth matching to a real depth is done analyzing of several calibration images, similar to matching pixels to planar coordinates. Tracer's XY coordinates are found on total focus images. Tracer's $\mathrm{Z}$ coordinate is taken from the depth map at XY position, in assumption that tracers do not overlap. "No overlap" assumption could be eliminated by using other reconstruction algorithm to reconstruct full 3D image instead of the total focus and the depth map. That algorithm is slower but has better accuracy, that is why we plan to use this algorithm in the future.
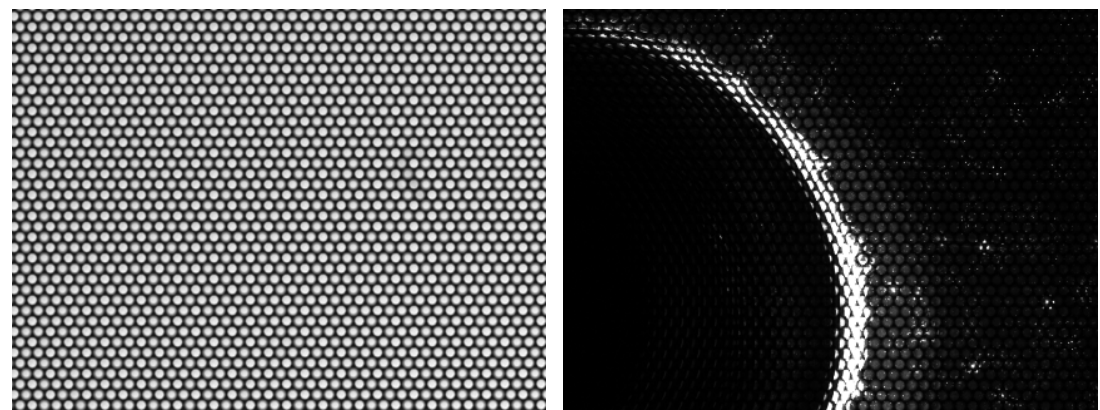

Fig. 1. Light field camera images: calibration or "white image" (left) and raw experimental image (right).

We used in-house software "ActualFlow" to process the acquired data in both LF-PTV and Tomo-PIV experiments. For these two cases 1000 particle pair input samples were analysed. A simple 3D-PTV algorithm with the nearest neigbour particle pairing and area search radius $20 \mathrm{px}$ was used to create $3 \mathrm{D}$ velocity fields on unstructured grids. Later 3DPTV velocity fields were used to build a mean velocity distribution on a regular grid with spatial resolution for a single vector $1.36 \times 1.36 \times 0.68 \mathrm{~mm}$ in order to have the same spatial resolution to compare the LF-PTV results with the tomographic measurements. Average number of samples used to calculate a single average velocity vector on the regular grid was near 500. It depended on the average number of particle pairs found for one 
instantaneous velocity field, which equaled to $\sim 1100$.

For Tomo-PIV the size of a reconstructed $3 \mathrm{D}$ object was $640 \times 824 \times 77$ voxels. A selfcalibration procedure was done to align all camera models directly using experimental particle images to get perfect multiple ray correspondence throughout the measurement volume. The tomographic reconstruction was done by SMART algorithm [6]. Correlation analysis was performed using the iterative multigrid algorithm with continuous interrogation box shifting. The total number of iterations was four: two steps were performed with a resolution of $64 \times 64 \times 32$ voxels and two extra iterations with a final resolution of $32 \times 32 \times 16$ voxels. The grid-overlapping factor was set to $75 \%$; therefore, the final correlation domain size for the calculation of a single velocity vector was $1.36 \times 1.36 \times 0.68 \mathrm{~mm}^{3}$.

\section{Experiment setup}

The experimental setup consisted of hydrodynamic contour, including a tank, a pump, flow meter, and measurement section. The measurement section was a narrow channel with length $390 \mathrm{~mm}$, width $200 \mathrm{~mm}$ and depth $\mathrm{h}=4 \mathrm{~mm}$, inside which the cylinder was installed. The cylinder with diameter $\mathrm{D}=10 \mathrm{~mm}$ and width $\mathrm{h}=4 \mathrm{~mm}$ was located on distance $14 \mathrm{D}$ from honeycomb on the axis of the channel (see Fig.2). The data were acquired for the Reynolds numbers $\operatorname{Re}=D U_{0} / v=3500$, where $\mathrm{U}_{0}$ is the bulk flow velocity. Measurements were carried out in a zone 2.8 D×3.0 D×0.4 D, located behind cylinder. The single-camera LF-PTV and the four-camera Tomo-PIV [7] were used to obtain volumetric distributions of scattering light from tracers.

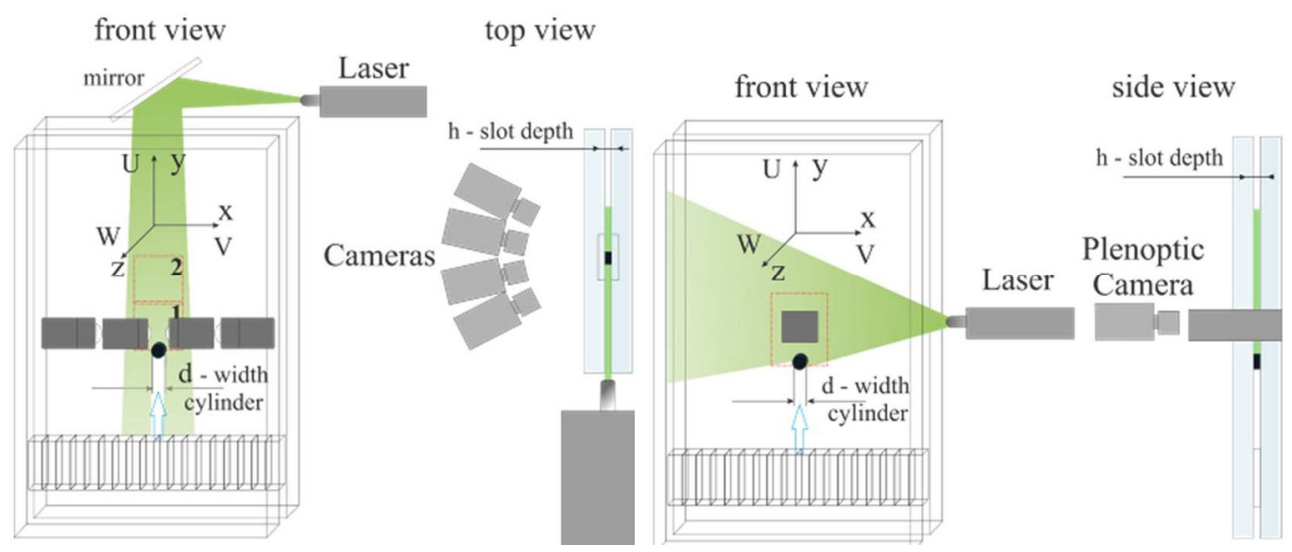

Fig. 2. Experimental setups: Tomo-PIV (left) and LF-PTV (right).

During LF-PTV measurements the system consisted of Nd:YAG laser EverGreen $(50 \mathrm{~mJ}, 532 \mathrm{~nm}, 15 \mathrm{~Hz})$, the light-field camera Raytrix (R11M-D-CL-A095-GS-B $4032 \times 2688$ pix $^{2}$, 9 um pixel size $192 \times 168$ microlenses, 24 pix diameter of microlens, $12 \mathrm{bit}, 10 \mathrm{~Hz}$ at full resolution) with the lens Carl Zeiss Makro-Planar 2/100 ZF.2, and synchronizing device Berkeley Nucleonics BNC 575. Particle image concentration for LFPTV during experiment was maintained at a level $0.003 \mathrm{ppp}$. Frame separation time was set at 300 us in order to keep the maximum particle image displacement less than $20 \mathrm{px}$.

In the case of Tomo-PIV experiment the measurement system consisted of a high-speed Nd:YLF New wave Pegasus (50 mJ, $527 \mathrm{~nm}$, at $1 \mathrm{kHz}$ ) laser, four high-speed PCO.1200 hs $\left(1024 \times 1280\right.$ pix $^{2}, 12 \mathrm{um}$ pixel size, $10 \mathrm{bit}, 636 \mathrm{~Hz}$ at full resolution) cameras and Polis synchronizing device. We used SIGMA AF $50 \mathrm{~mm}$ f/2.8 EX DG MACRO lenses for the optical setup. The cameras were installed at horizontal plane along circle with center 
located in the same place as the center of the cylinder. The thickness of the laser sheet was $4 \mathrm{~mm}$ in both cases. Mean particle image density at the projections was kept at $0.025 \mathrm{ppp}$. Frame separation time in this case was 1350 us.

\section{Results and conclusion}

A comparison between results of LF-PTV and Tomo-PIV was carried out in the present work. Well qualitative agreement was observed for the 3D structure of the flow in terms of distributions of longitudinal and transverse components of mean velocity. They are shown in the form of 3D isosurfaces and the cross-stream sections (see Fig 3, 4). In spite of the difference in particle image concentration, which affects the number of averaged samples to produce a single mean velocity vector for LF-PTV, quantitative comparisons between the velocity profiles of the longitudinal velocity carried out in the central plane showed good agreement. These results show great potential for volumetric PIV measurements using a single light field camera.

Phantom particles did not have significant influence in LF-PTV measurements, due to presence of several copies of particle images, corresponding to a real tracer, registered by the light field camera. The used algorithm assumed sharp texture, which was the case in the experiment. We expect better accuracy could be achieved by adapting for tracer features during reconstruction process: Gaussian intensity distributions on a black background.

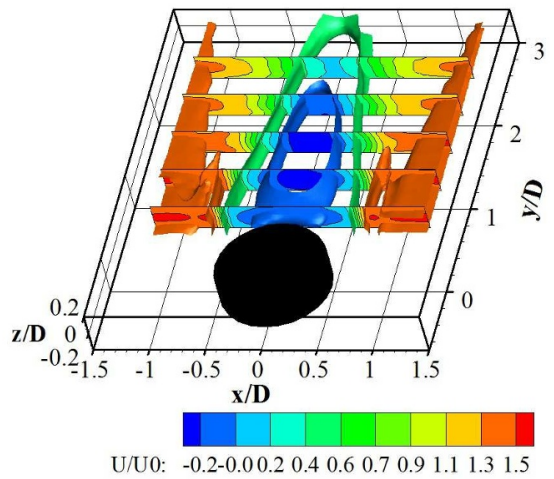

U/U0: $-0.2-0.00 .20 .40 .60 .70 .91 .11 .31 .5$

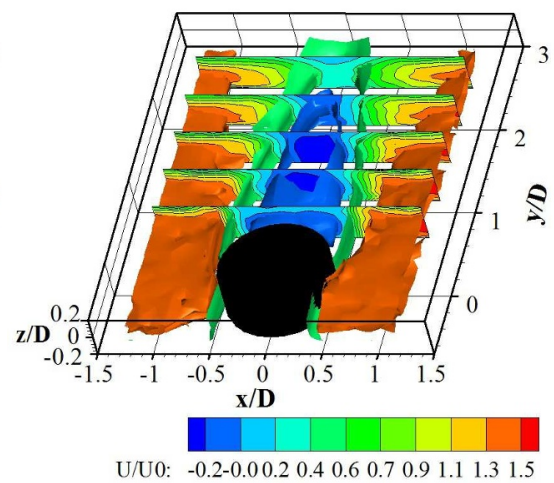

U/U0: $-0.2-0.00 .20 .40 .60 .70 .91 .11 .31 .5$

Fig. 3. Comparison of normalized longitudinal mean velocity Tomo-PIV (left) and LF-PTV (right).

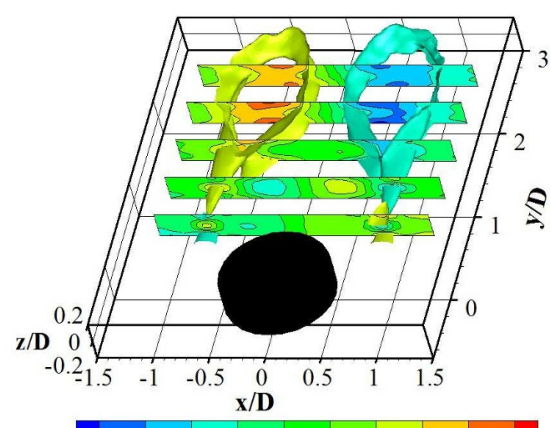

V/U0: $-0.26-0.20-0.14-0.09-0.030 .030 .090 .140 .200 .26$

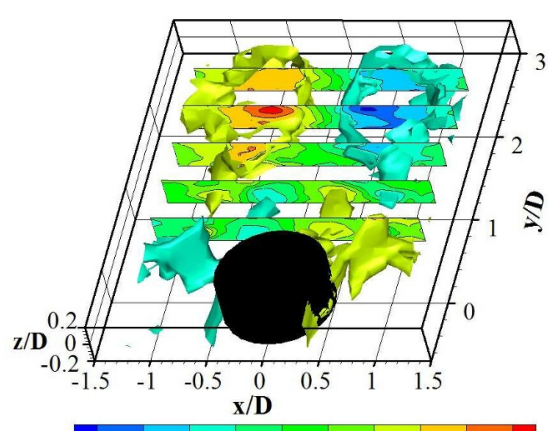

V/U0: $\quad-0.26-0.20-0.14-0.09-0.030 .030 .090 .140 .200 .26$

Fig. 4. Comparison of normalized transverse mean velocity Tomo-PIV (left) and LF-PTV (right). 
This work was funded by the Russian Foundation For Basic Research grant No. 18-38-00943. Experimental facilities of the work were partially supported by FASO Russia.

\section{References}

1. T.W. Fahringer, B.S. Thurow, Measurement Science and Technology, 29075202 (2018)

2. E. Hall, D. Guildenbecher, B. Thurow, Optics express, 25:18 (2017)

3. S. Shi, J. Ding, C. Atkinson, J. Soria, T.H. New, Experiments in Fluids, 59:46 (2018)

4. S Shi, J. Ding, T.H. New, J. Soria, Experiments in Fluids, 58:78 (2017)

5. A.V. Seredkin, M.V. Shestakov, M.P. Tokarev, AIP Conference Proceedings 1770, 030025 (2016)

6. A.V. Bilsky, V.A. Lozhkin, D.M. Markovich, M.P. Tokarev, Measurement Science and Technology, 24. P. 045301 (2013).

7. G.E. Elsinga, F. Scarano, Wieneke, B.W. van Oudheusden, Experiments in Fluids, 41. 933-947 (2006) 